Article

\title{
Symmetry Analysis and Conservation Laws of a Generalization of the Kelvin-Voigt Viscoelasticity Equation
}

\author{
Almudena P. Márquez * (D) and María S. Bruzón * \\ Department of Mathematics, University of Cadiz, Facultad de Ciencias Campus del Río San Pedro, \\ 11510 Puerto Real, Spain \\ * Correspondence: almudena.marquez@uca.es (A.P.M.); m.bruzon@uca.es (M.S.B.)
}

Received: 30 May 2019; Accepted: 24 June 2019; Published: 28 June 2019

\begin{abstract}
In this paper, we study a generalization of the well-known Kelvin-Voigt viscoelasticity equation describing the mechanical behaviour of viscoelasticity. We perform a Lie symmetry analysis. Hence, we obtain the Lie point symmetries of the equation, allowing us to transform the partial differential equation into an ordinary differential equation by using the symmetry reductions. Furthermore, we determine the conservation laws of this equation by applying the multiplier method.
\end{abstract}

Keywords: viscoelasticity; Kelvin-Voigt equation; Lie symmetries; optimal system; group-invariant solutions; conservation laws; multiplier method

\section{Introduction}

The continuous development of mechanics and its engineering applications have increased remarkably the interest in non-linear phenomena, such as viscoelasticity. Viscoelastic materials are of interest in a wide variety of applications, from passive damping to aircraft tire construction. A good modelling of the material's behaviour is essential for the accurate design incorporating this material.

Viscoelastic behaviour appears in materials showing some sort of liquid-like elastic behaviour. However, a simple Hooke's law linear elastic constitutive relationship is not an accurate representation of viscoelastic material's behaviour. Viscoelastic materials are commonly said to have "memory" because of their rheological properties.

The rheological models, such as the Kelvin-Voigt model or the Maxwell model, are usually used to describe the viscoelastic behaviour. The Kelvin-Voigt model consists of a lumped parameter model similar to a spring and dashpot in parallel, while the Maxwell model describes a serially connected spring and dashpot. In addition, many papers have been published studying these models [1-4].

Many physical phenomena, as viscoelasticity, are described by non-linear partial differential equations (PDEs). In particular, the Kelvin-Voigt viscoelasticity equation is given by

$$
u_{t t}-\left(C(x) u_{x}\right)_{x}-\left(B(x) u_{t x}\right)_{x}=0
$$

Nevertheless, in this paper we focus on a generalization of the Kelvin-Voigt viscoelasticity equation described by

$$
u_{t t}-\left(C(x) f(u)_{x}\right)_{x}-\left(B(x) u_{t x}\right)_{x}=0, \quad(t, x) \in \mathbb{R} \times \Omega,
$$

where $\Omega$ is an open subset of $\mathbb{R}$ and $u$ a scalar real-valued function. Also, $f(u)$ is a smooth enough non-linear function, and $C(x) \neq 0, B(x) \neq 0$ are smooth enough functions too, depending on the variable $x \in \Omega$. Throughout the paper the subscripts denote partial derivatives. 
There is no general theory for solving non-linear PDEs. Therefore, in this work, we use Lie theory to analyse Equation (1). Lie group analysis is a powerful tool to find general solutions for PDEs. This theory, originally defined by Sophus Lie at the end of the nineteenth century, develops solutions for PDEs by the transformation groups of Lie [5-8]. The fundamental basis of the Lie group method is that if a differential equation is invariant under a Lie group of transformations, then a reduction transformation exists. For instance, for PDEs with two independent variables like Equation (1), a single group reduction can transform the PDE into an ordinary differential equation (ODE), easier to solve.

Furthermore, a very important concept in the analysis of PDEs is the notion of conservation law. Conservation laws determine conserved quantities and constants of motion. They also detect integrability and check accuracy of numerical solutions method. Recently, Anco and Bluman $[9,10]$ developed a method that does not need the existence of Lagrangians because it is based on adjoint equations for non-linear equations and avoids the integrals of functions. This method called the multiplier method allows finding all local conservation laws admitted by any evolution equation. Many papers have been published in the last few years using this method [11-19].

The paper is organized as follows: In Section 2 we determine the Lie point symmetries of Equation (1). Then, in Section 3 we use the Lie point symmetries admitted by Equation (1) to obtain an optimal system of one-dimensional subalgebras. Afterwards, in Section 4 we find symmetry reductions for the one-dimensional subalgebras calculated previously. These reductions allow us to transform Equation (1) into an ODE. In Section 5 we derive the conservation laws of Equation (1) by applying the multiplier method. Finally, in Section 6 some conclusions are presented.

\section{Lie Point Symmetries}

A one-parameter group of infinitesimal transformations in $(x, t, u)$ is given by

$$
\begin{aligned}
x^{*} & =x+\varepsilon \xi(x, t, u)+\mathcal{O}\left(\varepsilon^{2}\right), \\
t^{*} & =t+\varepsilon \tau(x, t, u)+\mathcal{O}\left(\varepsilon^{2}\right), \\
u^{*} & =u+\varepsilon \phi(x, t, u)+\mathcal{O}\left(\varepsilon^{2}\right),
\end{aligned}
$$

where $\varepsilon$ is the group parameter and $\xi(x, t, u), \tau(x, t, u)$, and $\phi(x, t, u)$ are the infinitesimals.

Definition 1. A vector field

$$
X=\xi(x, t, u) \frac{\partial}{\partial x}+\tau(x, t, u) \frac{\partial}{\partial t}+\phi(x, t, u) \frac{\partial}{\partial u},
$$

where $\xi(x, t, u), \tau(x, t, u)$, and $\phi(x, t, u)$ are the infinitesimals, is a generator of a Lie point symmetry of Equation (1) if

$$
X^{(3)}\left(u_{t t}-\left(C(x) f(u)_{x}\right)_{x}-\left(B(x) u_{t x}\right)_{x}\right)=0,
$$

where $X^{(3)}$ is the third prolongation of the vector field (2) defined by

$$
X^{(3)}=X+\zeta_{x} \frac{\partial}{\partial u_{x}}+\zeta_{t} \frac{\partial}{\partial u_{t}}+\zeta_{x x} \frac{\partial}{\partial u_{x x}}+\zeta_{x t} \frac{\partial}{\partial u_{x t}}+\zeta_{t t} \frac{\partial}{\partial u_{t t}}+\zeta_{x x t} \frac{\partial}{\partial u_{x x t}},
$$


where the coefficients $\zeta_{x}, \zeta_{t}, \zeta_{x x}, \zeta_{x t}, \zeta_{t t}, \zeta_{x x t}$ are given by

$$
\begin{aligned}
\zeta_{x} & =D_{x} \phi-u_{t} D_{x} \tau-u_{x} D_{x} \xi \\
\zeta_{t} & =D_{t} \phi-u_{t} D_{t} \tau-u_{x} D_{t} \xi \\
\zeta_{x x} & =D_{x}\left(\zeta_{x}\right)-u_{x t} D_{x} \tau-u_{x x} D_{x} \xi \\
\zeta_{x t} & =D_{t}\left(\zeta_{x}\right)-u_{x t} D_{x} \tau-u_{x x} D_{t} \xi \\
\zeta_{t t} & =D_{t}\left(\zeta_{t}\right)-u_{t t} D_{t} \tau-u_{x t} D_{t} \xi \\
\zeta_{x x t} & =D_{x}\left(\zeta_{x t}\right)-u_{t t x} D_{x} \tau-u_{x x t} D_{x} \xi .
\end{aligned}
$$

Here $D_{i}$ stands for the total derivative operator.

Theorem 1. The Lie point symmetries of the generalization of the Kelvin-Voigt Equation (1), with $f(u)$ non-linear function, and $C(x) \neq 0, B(x) \neq 0$ arbitrary functions, are generated by the operator

$$
X_{1}=\partial_{t}
$$

For some particular functions of $f(u), C(x), B(x)$, there are additional generators given below.

1. If $f(u)$ is an arbitrary function, $C(x)=c_{1}$ and $B(x)=b_{1}$, with $c_{1}, b_{1}$ arbitrary constants,

$$
X_{2}^{1}=\partial_{x}
$$

2. If $f(u)$ is an arbitrary function, $C(x)=c_{1}$ and $B(x)=b_{1} x+b_{2}$, with $c_{1}, b_{1} \neq 0, b_{2}$ arbitrary constants,

$$
X_{2}^{2}=\left(b_{1} x+b_{2}\right) \partial_{x}+b_{1} t \partial_{t} .
$$

3. If $f(u)$ is an arbitrary function, $C(x)=\frac{4}{\left(n x+c_{1}\right)^{2}}$ and $B(x)=b_{1}$, with $c_{1}$, $b_{1}$ arbitrary constants, and $n$ a positive integer,

$$
X_{2}^{3}=\left(n x+c_{1}\right) \partial_{x}+2 n t \partial_{t} .
$$

4. If $f(u)$ is an arbitrary function, $C(x)=c_{2}\left(c_{1}-x\right)^{n}$ and $B(x)=\frac{\left(-c_{1}+x\right) c_{1} \sqrt{\left(c_{1}-x\right)^{n} c_{2}}}{n}$, with $c_{1}, c_{2}$ arbitrary constants, and $n$ a positive integer,

$$
X_{2}^{4}=\left(c_{1}-x\right) \partial_{x}+\frac{1}{2}(n-2) t \partial_{t}
$$

5. If $f(u)=\frac{f_{0}^{2} e^{\frac{-k k^{k}}{k}-1}\left(\frac{\left(k^{k}-1\right) u}{f_{0}}+\frac{f_{1} e^{k}-f_{1}}{f_{0}}\right)^{\frac{e^{k}}{e^{k}-1}+1}+2 f_{2} e^{k}-f_{2}}{2 e^{k}-1}, C(x)=c_{1}$ and $B(x)=b_{1}$, with $k$ a positive integer, $f_{0}, f_{1}, f_{2}$ positive constants, and $c_{1}, b_{1}$ arbitrary constants, besides $X_{2}^{5}=X_{2}^{1}$,

$$
X_{3}^{5}=x \partial_{x}+2 t \partial_{t}+2\left(-1+e^{-k}\right)\left(u+f_{1}\right) \partial_{u} .
$$

6. If $f(u)=\frac{f_{1}^{2} e^{\frac{-f_{0} f_{0}}{e^{f_{0}}-1}}\left(\frac{\left(e^{f_{0}}-1\right) u}{f_{1}}+\frac{f_{2} e^{f_{0}}-f_{2}}{f_{1}}\right)^{\frac{e^{f_{0}}}{e_{0}}+1}+1}{\left(e^{f_{0}}-1\right)\left(\frac{e^{f_{0}}}{e^{f_{0}}-1}+1\right)}+f_{3}, C(x)=c_{2}\left(c_{1}-x\right)^{n}$ and $B(x)=b_{1}$, with $f_{0}, f_{1}, f_{2}$, $f_{3}$ positive constants, $c_{1}, c_{2}, b_{1}$ arbitrary constants, and $n$ a positive integer,

$$
X_{2}^{6}=\left(c_{1}-x\right) \partial_{x}-2 t \partial_{t}-\left(1+e^{-f_{0}}\right)\left(u+f_{2}\right)(n+2) \partial_{u} .
$$


7. If $f(u)=\frac{f_{0} e^{\frac{-k k^{k}}{k}-1}\left(\frac{\left(e^{k}-1\right)\left(u+f_{1}\right)}{f_{0}}\right)^{\frac{2 e^{k}-1}{e^{k}-1}}+2 f_{2} e^{k}-f_{2}}{2 e^{k}-1}, C(x)=c_{2}\left(b_{2}-x\right)^{-m}$ and $B(x)=b_{3}\left(b_{2}-x\right)^{n}$, with $k a$ positive integer, $f_{0}, f_{1}, f_{2}$ positive constants, $b_{2}, b_{3}$ arbitrary constants, and $n, m$ positive integers,

$$
X_{2}^{7}=\left(b_{2}-x\right) \partial_{x}+t(n-2) \partial_{t}+\left(-1+e^{-k}\right)\left(u+f_{1}\right)(m+2 n-2) \partial_{u} .
$$

Proof of Theorem 1. Expanding (3), we obtain an overdetermined system satisfying the determining equations for the symmetry group. From $B^{2}\left(\tau_{u}\right)=0$ and $B^{2}\left(\tau_{x}\right)=0$, we find that $\tau$ depends only on $t$. The equations $B^{2}\left(\xi_{t}\right)=0$ and $B^{2}\left(\xi_{u}\right)=0$ reveal that $\xi$ is a function of $x$ alone. The remaining determining equations are

$$
\begin{aligned}
B^{2}\left(\xi_{u u}\right)=0, B^{2}\left(\xi_{t u}\right)=0, B^{2}\left(\tau_{u u}\right)=0, B\left(\tau_{u u}\right)=0, B^{2}\left(\tau_{u u u}\right)=0, B^{2}\left(\xi_{u u u}\right)=0, & =0, \\
B\left(2\left(\tau_{u x}\right) B-\xi_{u}\right) & =0, \\
B\left(2\left(\tau_{u u x}\right) B-\xi_{u u}\right) & =0, \\
B\left(\left(\tau_{u x}\right) B+\xi_{u}\right) & =0, \\
B\left(\left(\tau_{u x x}\right) B+\phi_{u u}-2\left(\tau_{t u}\right)\right) & =0, \\
B\left(\left(\xi_{u}\right)\left(f_{u u}\right) C-\left(\xi_{u u}\right)\left(f_{u}\right) C-\left(\xi_{t u u}\right) B\right) & =0, \\
B\left(f_{u}\left(\phi_{x x}\right) C+\left(\phi_{t x x}\right) B-\phi_{t t}\right) & =0, \\
B\left(\left(\tau_{u}\right)\left(f_{u}\right) C+\left(\phi_{u u}\right) B-\left(\tau_{t u}\right) B-2\left(\xi_{u x}\right) B\right) & =0, \\
B\left(\left(\tau_{u}\right)\left(f_{u}\right) C-\left(\phi_{u u}\right) B+\left(\tau_{t u}\right) B+2\left(\xi_{u x}\right) B\right) & =0, \\
B\left(\left(\tau_{u u}\right)\left(f_{u}\right) C-\left(\phi_{u u u u}\right) B+\left(\tau_{t u u}\right) B+2\left(\xi_{u u x}\right) B\right) & =0, \\
\xi\left(B^{\prime}\right)-\left(\tau_{x x}\right) B^{2}+\left(\tau_{t}\right) B-2\left(\xi_{x} B\right) & =0, \\
B\left(2\left(\tau_{x}\right)\left(f_{u}\right) C-2\left(\phi_{u x}\right) B+2\left(\tau_{t x}\right) B+\left(\xi_{x x}\right) B-2\left(\xi_{t}\right)\right) & =0, \\
B\left(\left(\tau_{x x}\right)\left(f_{u}\right) C-\left(\phi_{u x x}\right) B+\left(\tau_{t x x}\right) B+2\left(\phi_{t u}\right)-\tau_{t t}\right) & =0, \\
\xi\left(f_{u}\right) B\left(C^{\prime}\right)-\xi_{u}\left(B^{\prime}\right) C+\left(\left(f_{u u u}\right)\right) \phi B C+\left(\tau_{t}\right)\left(f_{u}\right) B C+\left(\phi_{t u}\right) B^{2}-2\left(\xi_{t x}\right) B^{2} & =0, \\
B\left(2\left(\tau_{x}\right)\left(f_{u u}\right) C+2\left(\tau_{u x}\right)\left(f_{u}\right) C-2\left(\phi_{u u x}\right) B+2\left(\tau_{t u x}\right) B+\left(\xi_{u x x}\right) B-2\left(\xi_{t u}\right)\right) & =0, \\
B\left(2\left(f_{u u}\right)\left(\phi_{x}\right) C+2 f_{u}\left(\phi_{u x}\right) C-\left(\xi_{x x}\right)\left(f_{u}\right) C+2\left(\phi_{t u x}\right) B-\left(\xi_{t x x}\right) B+\xi_{t t}\right) & =0, \\
\xi\left(f_{u u}\right) B\left(C^{\prime}\right)-\xi\left(f_{u u}\right)\left(B^{\prime}\right) C+f_{u}\left(\phi_{u u u}\right) B C+\left(f_{u u}\right)\left(\phi_{u}\right) B C+\left(\left(f_{u u u}\right)\right) \phi B C+\left(\tau_{t}\right)\left(f_{u u}\right) B C & \\
-2\left(\xi_{u x}\right)\left(f_{u}\right) B C+\left(\phi_{t u u}\right) B^{2}-2\left(\xi_{t u x}\right) B^{2} & =0 .
\end{aligned}
$$

Solving this system of equations we find the infinitesimals $\xi, \tau$ and $\phi$ of (2).

\section{Optimal Systems}

It is important to classify invariant solutions according to the classification of the associated symmetry generators. Then, one generator from each class is used to determine the desired set of invariant solutions. An optimal system of generators is defined as a set consisting of exactly one generator from each class [20].

The problem of obtaining an optimal system of subgroups is equivalent to that of obtaining an optimal system of subalgebras, and so we concentrate on the latter. For one-dimensional subalgebras, this classification problem is essentially equal to classifying the orbits of the adjoint representation [5].

The most important operator on vector fields is their Lie bracket or commutator. If $X_{i}$ and $X_{j}$ are vector fields, then their Lie bracket $\left[X_{i}, X_{j}\right]$ is the unique vector field satisfying

$$
\left[X_{i}, X_{j}\right]=X_{i}\left(X_{j}\right)-X_{j}\left(X_{i}\right) .
$$

The commutator table for the Lie algebra of Case 5 of Theorem (1) is shown in Table 1 . The $(i, j)$-th entry of the table expresses the Lie bracket $\left[X_{i}, X_{j}\right]$, for $i, j=1,2,3$. 
Table 1. The commutator table for Case 5 of Theorem (1).

\begin{tabular}{c|ccc}
\hline$\left[\boldsymbol{X}_{i}, \boldsymbol{X}_{j}\right]$ & $\boldsymbol{X}_{\mathbf{1}}$ & $\boldsymbol{X}_{2}^{5}$ & $\boldsymbol{X}_{3}^{5}$ \\
\hline$X_{1}$ & 0 & 0 & $2 X_{1}$ \\
$X_{2}^{5}$ & 0 & 0 & $X_{2}^{5}$ \\
$X_{3}^{5}$ & $-2 X_{1}$ & $-X_{2}^{5}$ & 0 \\
\hline
\end{tabular}

The adjoint representation can be constructed by summing the Lie series

$$
\begin{aligned}
\operatorname{Ad}\left(\exp \left(\epsilon X_{i}\right)\right) X_{j} & =\sum_{n=0}^{\infty} \frac{\epsilon^{n}}{n !}\left(\operatorname{ad} X_{i}\right)^{n}\left(X_{j}\right), \\
& =X_{j}-\epsilon\left[X_{i}, X_{j}\right]+\frac{\epsilon^{2}}{2}\left[X_{i},\left[X_{i}, X_{j}\right]\right]-\cdots
\end{aligned}
$$

To compute the adjoint representation, we use the Lie series (4) in conjunction with the commutator table in Table 1 . The adjoint table of this Lie algebra is shown in Table 2, with the $(i, j)$-th entry indicating $\operatorname{Ad}\left(\exp \left(\epsilon X_{i}\right)\right) X_{j}$.

Table 2. The adjoint table for Case 5 of Theorem (1).

\begin{tabular}{c|ccc}
\hline$A d\left(\exp \left(\epsilon X_{i}\right)\right) X_{j}$ & $X_{1}$ & $X_{2}^{5}$ & $X_{3}^{5}$ \\
\hline$X_{1}$ & $X_{1}$ & $X_{2}^{5}$ & $X_{3}^{5}-2 \epsilon X_{1}$ \\
$X_{2}^{5}$ & $X_{1}$ & $X_{2}^{5}$ & $X_{3}^{5}-\epsilon X_{2}^{5}$ \\
$X_{3}^{5}$ & $e^{2 \epsilon} X_{1}$ & $e^{\epsilon} X_{2}^{5}$ & $X_{3}^{5}$ \\
\hline
\end{tabular}

Theorem 2. A one-dimensional optimal system for the generalization of the Kelvin-Voigt Equation (1) is given by

$$
\lambda X_{1}+\mu X_{2}^{1}, \quad X_{2}^{2}, \quad X_{2}^{3}, \quad X_{2}^{4}, \quad X_{3}^{5}, \quad X_{2}^{6}, \quad X_{2}^{7},
$$

where $\lambda$ and $\mu$ are arbitrary constants.

Proof of Theorem 2. Let $\mathcal{G}$ be the symmetry algebra of Equation (1), with the adjoint representation for Case 5 in Theorem (1) determined in Table 2. Let $\mathrm{v}=a_{1} \mathrm{v}_{1}+a_{2} \mathrm{v}_{2}+a_{3} \mathrm{v}_{3}$ be a non-zero vector field of $\mathcal{G}$. For each case, we simplify the coefficients $a_{i}, i=1,2,3$, as much as possible through proper adjoints applications on $\mathrm{v}$.

\section{Symmetry Reductions}

In this section, we use the optimal system of one-dimensional subalgebras of Theorem (2) to determine the symmetry reductions of Equation (1).

The symmetry variables are found by solving the invariant surface condition

$$
\Phi \equiv \xi \partial_{x}+\tau \partial_{t}-\phi=0
$$

Reduction 1. From $\lambda X_{1}+\mu X_{2}^{1}$, we obtain the travelling wave reduction

$$
z=\mu x-\lambda t, \quad u=h(z),
$$

where $h(z)$ satisfies

$$
\lambda \mu^{2} b_{1} h^{\prime \prime \prime}-\mu^{2} c_{1} f^{\prime} h^{\prime \prime}+\lambda^{2} h^{\prime \prime}-\mu^{2} c_{1} f^{\prime \prime}\left(h^{\prime}\right)^{2}=0 .
$$

Reduction 2. From $X_{2}^{2}$, we obtain the invariant solution

$$
z=\frac{b_{1} x+b_{2}}{t}, \quad u=h(z)
$$


where $h(z)$ satisfies

$$
b_{1}^{2} h^{\prime \prime \prime} z^{2}+h^{\prime \prime} z^{2}+3 b_{1}^{2} h^{\prime \prime} z+2 h^{\prime} z+b_{1}^{2} h^{\prime}=0 .
$$

Reduction 3. From $X_{2}^{3}$, we obtain the invariant solution

$$
z=\frac{n x+c_{1}}{\sqrt{t}}, \quad u=h(z),
$$

where $h(z)$ satisfies

$$
2 b_{1} h^{\prime \prime \prime} n^{2} z+h^{\prime \prime} z^{2}+4 b_{1} h^{\prime \prime} n^{2}+3 h^{\prime} z=0 .
$$

Reduction 4. From $X_{2}^{4}$, we obtain the invariant solution

$$
z=-t^{\frac{2}{n-2}}\left(c_{1}-x\right), \quad u=h(z),
$$

where $h(z)$ satisfies

$$
\begin{aligned}
& 2 c_{1} \sqrt{c_{2}} h^{\prime \prime \prime} n(-z)^{\frac{n}{2}} z^{2}-4 c_{1} \sqrt{c_{2}} h^{\prime \prime \prime}(-z)^{\frac{n}{2}} z^{2}-4 h^{\prime \prime} n z^{2}+c_{1} \sqrt{c_{2}} h^{\prime \prime} n^{2}(-z)^{\frac{n}{2}} z \\
+ & 4 c_{1} \sqrt{c_{2}} h^{\prime \prime} n(-z)^{\frac{n}{2}} z-12 c_{1} \sqrt{c_{2}} h^{\prime \prime}(-z)^{\frac{n}{2}} z+2 h^{\prime} n^{2} z-8 h^{\prime} n z+c_{1} \sqrt{c_{2}} h^{\prime} n^{2}(-z)^{\frac{n}{2}} \\
- & 4 c_{1} \sqrt{c_{2}} h^{\prime}(-z)^{\frac{n}{2}}=0 .
\end{aligned}
$$

Reduction 5. From $X_{3}^{5}$, we obtain the invariant solution

$$
z=\frac{x}{\sqrt{t}}, \quad u=\frac{t^{-1+e^{-k}}}{h(z)}-f_{1}
$$

where $h(z)$ must satisfy a non-autonomous equation.

Reduction 6. From $X_{2}^{6}$, we obtain the invariant solution

$$
z=-\left(c_{1}-x\right) \sqrt{t}, \quad u=\frac{t^{\frac{\left(-1+e^{-} f_{0}\right)(n+2)}{2}}}{h(z)}-f_{2},
$$

where $h(z)$ must satisfy a non-autonomous equation.

Reduction 7. From $X_{2}^{7}$, we obtain the invariant solution

$$
z=-\frac{\left(b_{2}-x\right)}{t^{\frac{1}{n-2}}}, \quad u=\frac{t^{\frac{-\left(-1+e^{-k}\right)(m+2 n-2)}{n-2}}}{h(z)}-f_{1},
$$

where $h(z)$ must satisfy a non-autonomous equation.

The expressions of the reduced equations for $X_{3}^{5}, X_{2}^{6}$, and $X_{2}^{7}$ are omitted here to save space.

\section{Conservation Laws}

A local conservation law for the generalization of the Kelvin-Voigt viscoelasticity Equation (1) is a continuity equation

$$
D_{t} T+D_{x} X=0,
$$

holding for all solutions of Equation (1), where the conserved density $T$ and the spatial flux $X$ are functions of $x, t, u$, and derivatives of $u$. Here $D_{t}$ and $D_{x}$ denote total derivatives with respect to $t$ and $x$, respectively. The pair $(T, X)$ is called a conserved current.

Two local conservation laws are considered to be locally equivalent $[5,21]$ if they differ by a locally trivial conservation law $T=D_{x} \Theta, X=-D_{t} \Theta$, where $T$ and $X$ are evaluated on the set of solutions of Equation (1) and $\Theta$ is a function of $x, t, u$, and derivatives of $u$. 
A non-trivial conservation law can be written in a general form as

$$
\frac{d}{d t} \int_{\Omega} T d x=-\left.X\right|_{\partial \Omega^{\prime}}
$$

where $\Omega \subseteq \mathbb{R}$ is any fixed spatial domain. identity

Any local conservation law can be stated by using the characteristic form arising from a divergence

$$
D_{t} \tilde{T}+D_{x} \tilde{X}=\left(u_{t t}-\left(C(x) f(u)_{x}\right)_{x}-\left(B(x) u_{t x}\right)_{x}\right) Q,
$$

where $\tilde{T}=T+D_{x} \Theta$ and $\tilde{X}=X-D_{t} \Theta$ are locally equivalent to $T$ and $X$. The function $Q$ is called a multiplier. It satisfies

$$
Q=E_{u}(\tilde{T}),
$$

where $E_{u}$ represents the Euler operator with respect to $u$ [5], that is

$$
E_{u}=\partial_{u}-D_{x} \partial_{u_{x}}-D_{t} \partial_{u_{t}}+D_{x} D_{t} \partial_{u_{x t}}+D_{x}^{2} \partial_{u_{x x}}+\cdots
$$

For evolution equations, there is a one-to-one relationship between non-zero multipliers and non-trivial conserved current vectors up to local equivalence [5,9]. In general, a function $Q\left(x, t, u, u_{t}, u_{x}, \ldots\right)$ is a multiplier if it verifies that $\left(u_{t t}-\left(C(x) f(u)_{x}\right)_{x}-\left(B(x) u_{t x}\right)_{x}\right) Q$ is a divergence expression for all function $u(x, t)$. Given a multiplier $Q$, the conserved density can be determined by

$$
T=\int_{0}^{1} u Q\left(x, t, \lambda u, \lambda u_{x}, \lambda u_{x x}, \ldots\right) d \lambda .
$$

The divergence condition yields to the determining equation

$$
E_{u}\left(\left(u_{t t}-\left(C(x) f(u)_{x}\right)_{x}-\left(B(x) u_{t x}\right)_{x}\right) Q\right)=0 .
$$

In order to give a complete classification of multipliers, we write and split the determining Equation (6) with respect to the variables $u_{t t}, u_{t t t}, u_{t t x}, u_{t x x}, u_{x x x}, u_{t t t x}, u_{t t x x}, u_{t x x x}, u_{x x x x}$. Thus, we get a linear determining system for $Q\left(x, t, u, u_{t}, u_{x}, \ldots\right)$. The multipliers are found by solving the system with the same algorithmic method used for the determining equations for infinitesimal symmetries. Then, integrating the characteristic Equation (5) for each multiplier, we find the conserved current.

Theorem 3. The multipliers admitted by the generalization of the Kelvin-Voigt Equation (1), with $f(u) a$ smooth enough non-linear function, and $C(x) \neq 0, B(x) \neq 0$ smooth enough arbitrary functions, are given by

$$
Q_{1}=1, \quad Q_{2}=t, \quad Q_{3}=\int \frac{1}{C(x)} d x .
$$

Theorem 4. All non-trivial local conservation laws admitted by the generalization of the Kelvin-Voigt Equation (1), with $f(u)$ a smooth enough non-linear function, and $C(x) \neq 0, B(x) \neq 0$ smooth enough arbitrary functions, are given by

1. For the multiplier $Q_{1}=1$, the conserved density and the spatial flux are

$$
\begin{aligned}
T_{1} & =u_{t}, \\
X_{1} & =-B(x) u_{t x}-C(x) f(u)_{x} .
\end{aligned}
$$

2. For the multiplier $Q_{2}=t$, the conserved density and the spatial flux are

$$
\begin{aligned}
T_{2} & =t u_{t}-u, \\
X_{2} & =-t B(x) u_{t x}-t C(x) f(u)_{x} .
\end{aligned}
$$


3. For the multiplier $Q_{3}=\int \frac{1}{C(x)} d x$, the conserved density and the spatial flux are

$$
\begin{aligned}
T_{3} & =\left(\int \frac{1}{C(x)} d x\right) u_{t}+\left(\frac{B(x) C^{\prime}(x)}{C(x)^{2}}-\frac{B^{\prime}(x)}{C(x)}\right) u, \\
X_{3} & =-\left(\int \frac{1}{C(x)} d x\right) B(x) u_{t x}-\left(\int \frac{1}{C(x)} d x\right) C(x) f(u)_{x}+\frac{B(x) u_{t}}{C(x)}+f(u) .
\end{aligned}
$$

\section{Conclusions}

In this paper, we studied a generalization of the Kelvin-Voigt viscoelasticity equation given by the partial differential Equation (1). Firstly, we determined a complete Lie group classification. Then, we constructed the optimal system of one-dimensional subalgebras. These one-dimensional subalgebras have been used to find the symmetry reductions, allowing us to transform the partial differential equation into an ordinary differential equation. Moreover, we analysed all conservation laws for this equation by applying the multiplier method.

Author Contributions: A.P.M. and M.S.B. worked together in the derivation of the mathematical results. Both authors provided critical feedback and helped shape the research, analysis and manuscript.

Funding: This research received no external funding.

Acknowledgments: The authors express their sincere gratitude to the financial support of Junta de Andalucía FQM-201 group. We gratefully thank the reviewers for their assistance.

Conflicts of Interest: The authors declare no conflict of interest.

\section{Abbreviations}

The following abbreviations are used in this manuscript:

PDE Partial Differential Equation

ODE Ordinary Differential Equation

\section{References}

1. Atallah-Baraket, A.; Trabelsi, M. Analysis of the energy decay of a viscoelasticity type equation. Analele Stiintifice ale Universitatii Ovidius Constanta 2016, 24, 21-45. [CrossRef]

2. Schiessel, H.; Metzler, R.; Blumen, A.; Nonnenmacher, T.F. Generalized viscoelastic models: Their fractional equations with solutions. J. Phys. A: Math. Gen. 1995, 28, 6567. [CrossRef]

3. Lei, Y.; Adhikari, S.; Friswell, M.I. Vibration of nonlocal Kelvin-Voigt viscoelastic damped Timoshenko beams. Int. J. Eng. Sci. 2013, 66, 1-13. [CrossRef]

4. Lewandowski, R.; Chorazyczewski, B. Identification of the parameters of the Kelvin-Voigt and the Maxwell fractional models, used to modeling of viscoelastic dampers. Comput. Struct. 2010, 88, 1-17. [CrossRef]

5. Olver, P.J. Applications of Lie Groups to Differential Equations; Springer-Verlag: New York, NY, USA, 1986.

6. Bluman, G.W.; Anco, S.C. Symmetry and Integration Methods for Differential Equations; Springer-Verlag: New York, NY, USA, 2002.

7. Bluman, G.W.; Cheviakov, A.F.; Anco, S.C. Applications of Symmetry Methods to Partial Differential Equations; Springer-Verlag: New York, NY, USA, 2010.

8. Bluman, G.W.; Kumei, S. Symmetries and Differential Equations; Springer-Verlag: New York, NY, USA, 1989.

9. Anco, S.C.; Bluman, G.W. Direct constrution method for conservation laws of partial differential equations Part I: Examples of conservation law classifications. Eur. J. Appl. Math. 2002, 5, 545-566. [CrossRef]

10. Anco, S.C.; Bluman, G.W. Direct constrution method for conservation laws of partial differential equations Part 2: General treatment. Eur. J. Appl. Math. 2002, 5, 545-566. [CrossRef]

11. Bruzón, M.S.; Márquez, A.P. Conservation laws of one-dimensional strain-limiting viscoelasticity model. AIP Conf. Proc. 2017, 1836, 020081.

12. Bruzón, M.S.; Recio, E.; Garrido, T.M.; Márquez, A.P. Conservation laws, classical symmetries and exact solutions of the generalized KdV-Burguers-Kuramoto equation. Open Phys. 2017, 15, 433-439. [CrossRef] 
13. Bruzón, M.S.; Recio, E.; Garrido, T.M.; Márquez, A.P.; de la Rosa, R. On the similarity solutions and conservation laws of the Cooper-Shepard-Sodano equation. Math. Meth. Appl. Sci. 2018, 41, 7325-7332. [CrossRef]

14. Motsepa, T.; Khalique, C.M.; Gandarias, M.L. Symmetry Analysis and Conservation laws of the Zoomeron Equation. Symmetry 2017, 9, 27. [CrossRef]

15. Mothibi, D.M.; Khalique, C.M. Conservation laws and Exact Solutions of a Generalized Zakharov-Kuznetsov Equation. Symmetry 2015, 7, 949-961. [CrossRef]

16. Anco, S.C.; Rosa, M.; Gandarias, M.L. Conservation laws and symmetries of time-dependent generalized KdV equations. Discret. Contin. Dyn. Syst. Ser. S 2018, 11, 607-615. [CrossRef]

17. Gandarias, M.L.; Khalique, M. Symmetries, solutions and conservation laws of a class of nonlinear dispersive wave equations. Commun. Nonlinear Sci. Numer. Simul. 2016, 32, 114-131. [CrossRef]

18. de la Rosa, R.; Gandarias, M.L.; Bruzón, M.S. On symmetries and conservation laws of a Gardner equation involving arbitrary functions. Appl. Math. Comput. 2016, 290, 125-134. [CrossRef]

19. Bruzón, M.S.; Márquez, A.P.; Garrido, T.M.; Recio, E.; de la Rosa, R. Conservation laws for a generalized seventh order KdV equation. J. Comput. Appl. Math. 2019, 354, 682-688. [CrossRef]

20. Hydon, P.E. Symmetry Methods for Differential Equations: A Beginner's Guide; Cambridge University Press: Cambridge, UK, 2000.

21. Anco, S.C. Generalization of Noether's theorem in modern form to non-variational partial differential equations. In Recent Progress and Modern Challenges in Applied Mathematics, Modeling and Computational Science; Springer: New York, NY, USA, 2017; pp. 119-182.

(C) 2019 by the authors. Licensee MDPI, Basel, Switzerland. This article is an open access article distributed under the terms and conditions of the Creative Commons Attribution (CC BY) license (http:/ / creativecommons.org/licenses/by/4.0/). 